\title{
The Detection of the Upper Boundary of Breasts Using 4D Scanning Technology
}

\author{
Jie PEI *1, Linsey GRIFFIN ${ }^{2}$, Jintu FAN ${ }^{1,3}$, Susan P. ASHDOWN ${ }^{1}$ \\ ${ }^{1}$ Cornell University, Ithaca, NY, USA; \\ ${ }^{2}$ University of Minnesota, St Paul, MN, USA; \\ ${ }^{3}$ The Hong Kong Polytechnic University, Kowloon, Hong Kong \\ https://doi.org/10.15221/19.266
}

\begin{abstract}
Defining the boundary of female breasts is an unavoidable and very essential step before estimating the volume or surface area of the breast. Anthropometric measurements such as these are critical to the understanding of breast shape and the development of bra products. However, a non-contact method that accurately detects the boundary between breasts and chest wall, for breasts evaluated in the upright body position, has not been reported before. In this study, with the help of 4D body scanning and taking advantage of the time delay in the vertical displacement between the breasts and the chest wall during physical activity, we 4D-scanned 26 female participants using the Temporal 3dMD system and proposed a method to visualize the amount of variability for the relative displacement in the vertical direction, to facilitate the definition of the upper boundary of breasts. By viewing each breast as a cone or hemisphere, and processing the scan through vertical slicing, we were able to make all the scans having the same number of points $(7200=180 \times 40$. There were 40 vertical slices in total, and on each slice, there was one point located at every other degree from $-180^{\circ}$ to $180^{\circ}$ ) sorted in the exact same order. After proper alignment, we calculated the z-coordinate difference between a scan captured while the participant was running (there were nine dynamic scans that formed a gait cycle) and the corresponding scan of the same participant captured in static standing (the static scan). Then we were able to calculate the standard deviation (SD) value of the $z$-coordinate differences (i.e. the relative vertical displacements) across the nine dynamic scans. Heat maps were then created with the SD values mapped onto the 3D surface of the static scan and presented by gradient colors. This study is one of the first comprehensive studies that investigate the vertical displacement and the shape deformation of breasts during running using 4D scanning technology. Ultimately, the method and results can increase the understanding of breast kinematics, and benefit the product design of bras, especially for sports bras.
\end{abstract}

Keywords: 4D scanning; Female breasts; Breast boundary; Bra

\section{Introduction}

Defining the boundary of breasts is an unavoidable and very essential step before estimating the volume or surface area of the breast [1-6]. Anthropometric measurements such as these are critical to the understanding of breast shape and the development of bra products [7-9].

Multiple methods have been proposed to find the boundary of breasts for mammograms or for breast MR images [10-13], however, their focus is to separate breasts from the background (the ambient environment) rather than from the chest wall. Coltman, Steele and McGhee scanned their participants while they were laying in prone position, letting the breasts naturally suspend in a gap between two tables (similar to the standard posture for breast MRI) [6]. They defined the boundary of the breasts on the 3D scans in Geomagic ${ }^{\circledR}$ based on visual judgment. In fact, determining breast boundary for participants in the upright position is more challenging, especially for small chested females. Lee, Hong and Kim proposed a folding line method to find breast boundary for participants in the upright position [7]. Their method requires pushing the entire breast upward and inward by hand to reveal the skin folds, where the natural boundary locates. The method itself can be efficient, only that it involves hand contact on a sensitive body area, which may cause psychological discomfort for some participants. To the best of our knowledge, a non-contact method that facilitates the accurate detection of the boundary between breasts and chest wall, for breasts evaluated in the upright body position, has not been reported before. The development of $4 \mathrm{D}$ scanning technology introduces the fourth dimension, i.e. time, and makes it easier to acquire the boundary information. This is because during physical activities, the breasts usually have a time delay in displacement with the chest wall, and this relative displacement in vertical direction causes the bouncing of breasts $[8,14]$.

*ip2285@cornell.edu; +1 607-216-5913 
In this study, we 4D scanned 26 female participants using Temporal 3dMD system and proposed a method to visualize the extent of the relative displacement in the vertical direction to facilitate the definition of the upper boundary of breasts. Ultimately, the method can benefit the product design of bras, especially for sports bras.

\section{Methodology}

With approval obtained from IRB (Institutional Review Board), 26 female participants, who self-identified as wearing Size 18, were recruited for 4D body scanning, using a Temporal 3dMD full body scanner. Consents were obtained from all of the participants. A 4D scan is essentially a series of 3D scans captured in time while the participant was in motion. For each participant, we selected nine frames (or nine 3D body scans) while the participant was performing running-in-place. The scan captured while she was in standing position, with arms placed slightly apart from the torso, was also selected, as this is the standard scanning posture for 3D body scanning. The selected scans were processed in MeshLab and Meshmixer to remove the head and the limbs, leaving only the torso, and to fill holes if any [15]. After also removing the area below the swell of the breasts, the scans were processed in Matlabß to have exactly same number of points on the scan surface sorted in an organized fashion. The scans were processed to have vertical slices, and one breast was processed at a time. We assume that the shape of breast is quasi-hemispherical or quasi-conical. Based on this assumption, each slice along the height of the breast projection can be regarded as quasi-circular, which allows us to control the number of points on each slice. In addition, we set the location of the bust point (i.e. its $x$ and $z$ coordinates projected on any frontal plane) to be the origin for the quasi-circles. As shown in Figure 1, there are 40 vertical slices (sliced by equally spaced frontal planes) and 180 points on each slice (there is one point located at every 2 degrees from $-180^{\circ}$ to $180^{\circ}$, note that $-180^{\circ}$ and $180^{\circ}$ are essentially the same angle, corresponding to the same point, i.e. the beginning and the end of a circle). Therefore, the total number of points is $7200(180 \times 40)$.
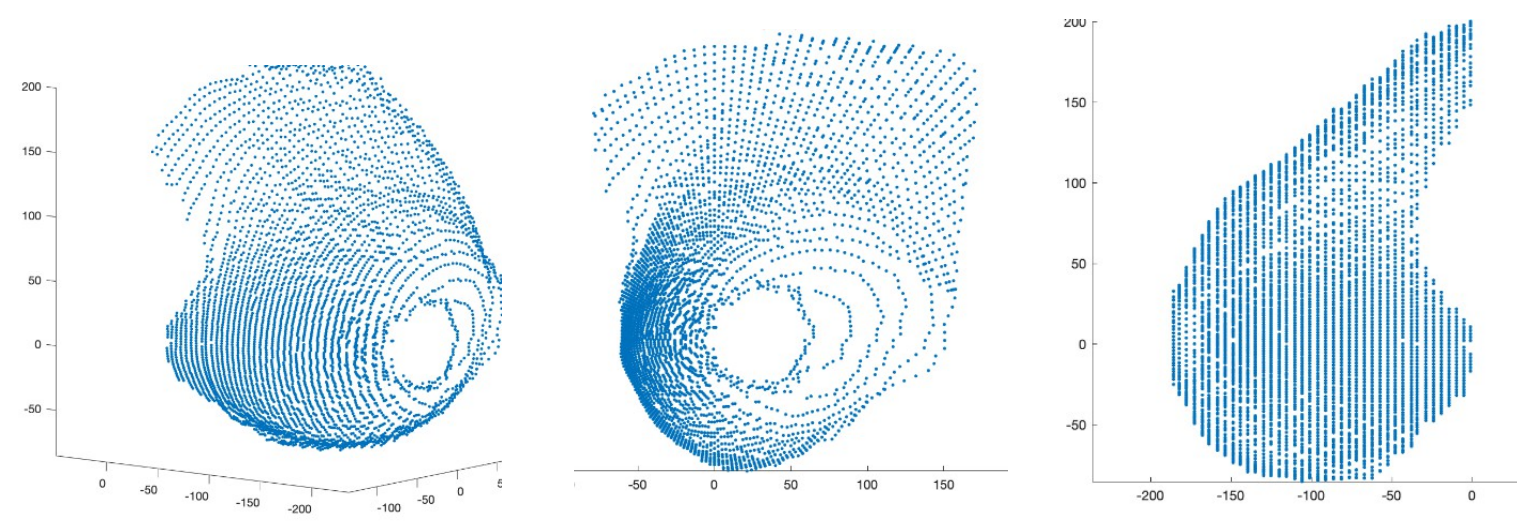

Figure 1. The processing of the scans in Matlab®

Then the nine scans captured during motion were plotted together with the scan (of the same participant) captured in static state, one at a time, and each of them was shifted and rotated manually to align with the static scan at the upper back area. Perfect alignment cannot be achieved, but the upper back area has relatively less change in shape, and therefore it is more ideal to be the benchmark for alignment (note that body landmarks are not ideal due to various reasons, see [15]).

Now that every scan had the same number of points, we were able to compare the z-coordinates of the corresponding points. The point-wise relative displacement in vertical direction between a scan captured during motion, and its corresponding scan captured in static state, can be estimated via Eq.1. To estimate the amount of variability of the relative displacements, we further calculated the standard deviation of those z-coordinate differences via Eq. 2.

$$
d_{j}=z_{i j}-z_{i 0}
$$

where $d_{j}$ is an array containing the vertical displacements of all the 7200 points for one of the nine scans captured during motion, i.e. the $\mathrm{j}$-th $\operatorname{scan}(1 \leq \mathrm{j} \leq 9), \quad z_{i j}$ is the $z$-coordinate of the $\mathrm{i}$-th point of that scan $(1 \leq \mathrm{i} \leq 7200)$, while $z_{i 0}$ is the $z$-coordinate of the $\mathrm{i}$-th point of the static scan (of the same participant) $(1 \leq \mathrm{i} \leq 7200)$. 


$$
S D=\sqrt{\frac{\sum\left(d_{j}-d_{\text {avg }}\right)^{2}}{n-1}}
$$

where SD represents the standard deviation of $d_{j}, d_{\text {avg }}$ is the mean value of $d_{j}$, and $\mathrm{n}=9$ is the total number of scans captured in motion state.

\section{Results and Discussions}

Heat maps were created with each SD value mapped onto the 3D surface of the static scan (i.e. the base scan). Figure 2 shows the heat maps for six randomly selected participants. The dark blue color corresponds to minimal variability in vertical displacement, whereas the dark red color corresponds to maximal variability in vertical displacement. For some participants, high SD (standard deviation) values can be observed at the armpit area and/or the shoulder area. This is most likely due to the arm swing rather than the breast tissue extending all the way to the armpit.
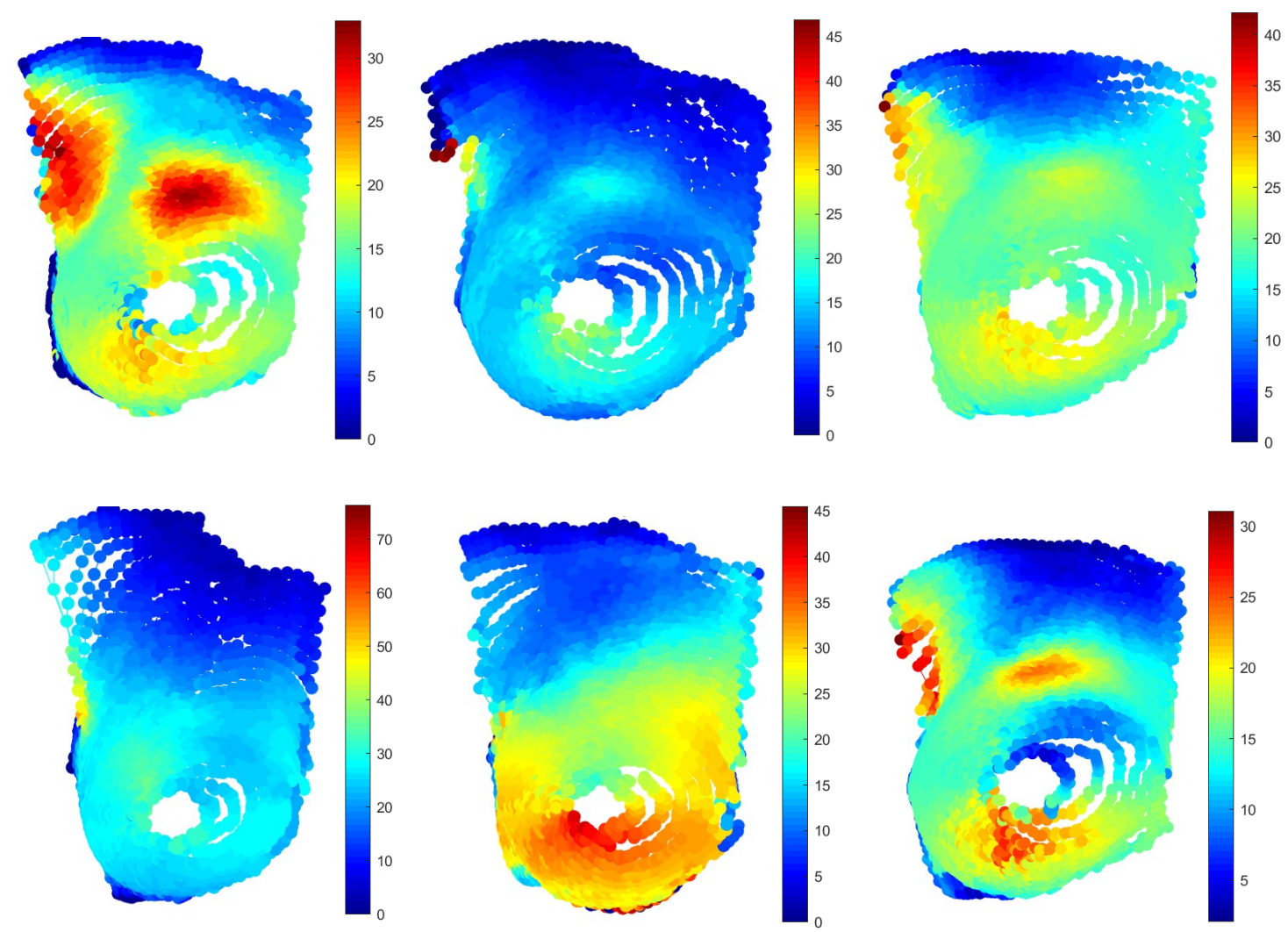

Figure 2. Heat map showing the amount of variability of the relative vertical displacements

The heat maps can provide helpful information to facilitate the definition of the boundary of breasts. Based on the calculated SD value of the relative vertical displacements, a threshold value was defined for each participant with the reference of the color patterns in her heat map. Points with SD values smaller than the threshold value (indicating less variability in the relative displacements) were identified, and mapped back to the original scan (where points are not sorted by slices and angles). Those points on the original scan and their neighboring points were highlighted in red, and the combination of them forms the area for deletion (see the first column in Table 1). Furthermore, we modified the area slightly (manually) to remove points at the armpit area, for it should not be considered as an extension of the breast (as explained earlier), to compensate the asymmetricity caused by running posture, to compensate the possible impact of the bra, and to make the boundary curves smooth (see the second column in Table 1). In the end, we obtained the 3D model of the breasts separated from the chest wall (the last column in Table 1). 
Table 1. Separation of the breasts from the chest wall

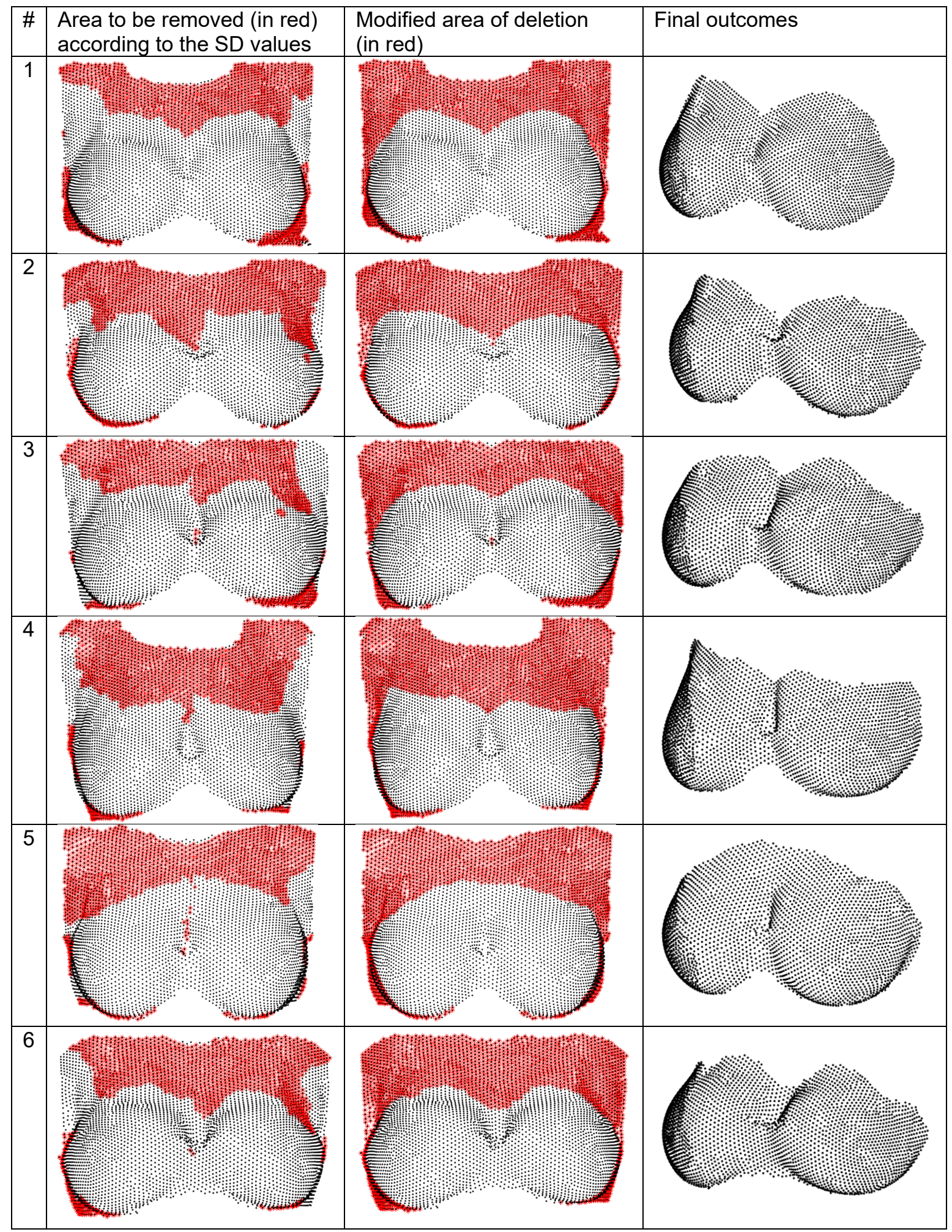

\section{Conclusions}

In this study, we 4D scanned 26 female participants using Temporal 3dMD system. Nine frames while the participant was performing running-in-place, and one frame while the participant was in standing posture were included. For each scan, the head, the limbs and the section below the swell of the breasts were removed. The remaining scan was processed in Matlab $₫$ to have exactly same number of points sorted in an organized fashion: by assuming the shape of the breast as quasi-hemispherical or quasi- 
conical and slicing it with vertical planes, we were able to quantify the vertical displacement of each breast.

The main goal of this paper is to define the upper boundary of breasts. To achieve this goal, we first computed the differences in z-coordinates between a scan captured during motion, and its corresponding scan captured in static. We then computed the standard deviation (SD) of those zcoordinate differences which represents the amount of variability of the relative vertical displacements. The breasts tissue has more variability in vertical displacement, compared with the none-breast-tissue area. Heat maps were created with the SD values mapped onto the 3D surface of the static scan (i.e. the base scan), and used as a reference to determine the threshold value for the separation of the breasts from the chest wall (in other words, to determine the boundary of the breasts). Furthermore, the boundary curves defined solely based on the SD values were modified slightly to: 1) remove points at the armpit area, for its high variability is more likely due to the impact of arm swing rather than the breast tissue extending all the way to the armpit, 2) to compensate the asymmetry caused by running posture, and 3 ) to compensate the possible impact of the bra that the participant was wearing.

There are several limitations in this study. Firstly, the participants were scanned while performing running-in-place, which may be different from actual running. However, our method to detect breast boundaries can work for any kind of physical activities, as long as it involves rapid up-and-down movement of the torso. Fewer frames may be included to generate the heat maps to reduce the amount of work for preparing the scans, sacrificing the accuracy of the results. Secondly, the way we processed scans does not guarantee the tracking of the same points on the actual skin surface across the various scans captured during motion. Lastly, our method still requires a manual modification on the boundary curves based on subjective judgements, for example, we tried to compensate the impact of the bra via this modification but the interaction between the breasts and the bra is not quantified nor thoroughly investigated. Future studies can 4D scan participants with and without wearing a bra to study the impact of bras on the shape change of breasts during motion.

\section{References}

[1] Smith, Jr DJ, Jr WE Palin, Victor L. Katch, and James E. Bennett. "Breast volume and anthropomorphic measurements: normal values." Plastic and reconstructive surgery 78 , no. 3 (1986): 331-335. https://doi.org/10.1097/00006534-198609000-00008

[2] Edsander-Nord, ĀSa, Marie Wickman, and GÖRan Jurell. "Measurement of breast volume with thermoplastic casts." Scandinavian journal of plastic and reconstructive surgery and hand surgery 30, no. 2 (1996): 129-132. https://doi.org/10.3109/02844319609056394

[3] Bulstrode, N., E. Bellamy, and S. Shrotria. "Breast volume assessment: comparing five different techniques." The Breast10, no. 2 (2001): 117-123. https://doi.org/10.1054/brst.2000.0196

[4] Kovacs, Laszlo, Maximilian Eder, Regina Hollweck, Alexander Zimmermann, Markus Settles, Armin Schneider, Matthias Endlich et al. "Comparison between breast volume measurement using 3D surface imaging and classical techniques." The Breast 16, no. 2 (2007): 137-145. https://doi.org/10.1016/.j.breast.2006.08.001

[5] Xi, Wenjing, Aurelia Trisliana Perdanasari, Yeesiang Ong, Sheng Han, Peiru Min, Weijie Su, Shaoqing Feng, Lucrezia Pacchioni, Yi Xin Zhang, and Davide Lazzeri. "Objective breast volume, shape and surface area assessment: a systematic review of breast measurement methods." Aesthetic plastic surgery 38, no. 6 (2014): 1116-1130. https://doi.org/10.1007/s00266014-0412-5

[6] Coltman, Celeste E., Julie R. Steele, and Deirdre E. McGhee. "Effects of age and body mass index on breast characteristics: a cluster analysis." Ergonomics 61, no. 9 (2018): 1232-1245. https://doi.org/10.1080/00140139.2018.1481229

[7] Lee, Hyun-Young, Kyunghi Hong, and Eun Ae Kim. "Measurement protocol of women's nude breasts using a 3D scanning technique." Applied Ergonomics 35, no. 4 (2004): 353-359. https://doi.org/10.1016/i.apergo.2004.03.004

[8] McGhee, Deirdre E., Julie R. Steele, William J. Zealey, and George J. Takacs. "Bra-breast forces generated in women with large breasts while standing and during treadmill running: Implications for sports bra design." Applied ergonomics 44, no. 1 (2013): 112-118. https://doi.org/10.1016/i.apergo.2012.05.006

[9] Coltman, Celeste E., Deirdre E. McGhee, and Julie R. Steele. "Three-dimensional scanning in women with large, ptotic breasts: implications for bra cup sizing and design." Ergonomics 60, no. 3 (2017): 439-445. https://doi.org/10.1080/00140139.2016.1176258 
[10] Ojala, T., J. Näppi, and O. Nevalainen. "Accurate segmentation of the breast region from digitized mammograms." Computerized Medical Imaging and Graphics25, no. 1 (2001): 47-59. https://doi.org/10.1016/S0895-6111(00)00036-7

[11] Ferrari, R. J., A. F. Frere, R. M. Rangayyan, J. E. L. Desautels, and R. A. Borges. "Identification of the breast boundary in mammograms using active contour models." Medical and Biological Engineering and Computing 42, no. 2 (2004): 201-208. https://doi.org/10.1007/BF02344632

[12] Giannini, Valentina, Anna Vignati, Lia Morra, Diego Persano, Davide Brizzi, Luca Carbonaro, Alberto Bert, Francesco Sardanelli, and Daniele Regge. "A fully automatic algorithm for segmentation of the breasts in DCE-MR images." In 2010 Annual International Conference of the IEEE Engineering in Medicine and Biology, pp. 3146-3149. IEEE, 2010. https://doi.org/10.1109/IEMBS.2010.5627191

[13] Kus, Pelin, and Irfan Karagoz. "Fully automated gradient based breast boundary detection for digitized X-ray mammograms." Computers in biology and medicine 42, no. 1 (2012): 75-82. https://doi.org/10.1016/i.compbiomed.2011.10.011

[14] Haake, Steve, and Joanna Scurr. "A dynamic model of the breast during exercise." Sports Engineering 12, no. 4 (2010): 189-197. https://doi.org/10.1007/s12283-010-0046-z

[15] Pei, J., Griffin, L., Ashdown, S. P. \& Fan, J. "The sudy of breast shape during running using 4D scanning technology." (2019+). In preparation. 